\title{
Civilisations
}

Revue internationale d'anthropologie et de sciences

humaines

40-1 | 1991

L'Asie et le Pacifique

\section{L'Asie du nord-est : incertitudes et perspectives}

\section{Philippe Debroux}

URL : http://journals.openedition.org/civilisations/1686

DOI : 10.4000/civilisations. 1686

ISSN : 2032-0442

\section{Éditeur}

Institut de sociologie de l'Université Libre de Bruxelles

\section{Édition imprimée}

Date de publication : 1 janvier 1992

Pagination : 189-209

ISBN : 2-87263-063-5

ISSN : 0009-8140

\section{Référence électronique}

Philippe Debroux, "L'Asie du nord-est : incertitudes et perspectives », Civilisations [En ligne], 40-1 | 1991, mis en ligne le 07 juillet 2009, consulté le 03 mai 2019. URL : http:// journals.openedition.org/civilisations/1686 ; DOI : 10.4000/civilisations.1686 


\title{
L'ASIE DU NORD-EST : INCERTITUDES ET PERSPECTIVES
}

\author{
Philippe DEBROUX
}

Introduction

Les régions de l'Asie de l'Est et du Sud-Est ont connu un développement économique extrêmement rapide ces vingt dernières années. Leurs économies deviennent intégrées horizontalement. Le commerce intra-asiatique est en croissance rapide de même que les flux d'investissements régionaux, non plus seulement du Japon mais aussi de Taiwan et de la Corée du Sud. De grands projets de coopération régionale et sous-régionale sont discutés tels ceux d'une voiture entièrement fabriquée dans la région ou d'une compagnie aérienne commune. Sur un plan institutionnel, beaucoup d'idées sont discutées de Canberra à Manille : sur la désirabilité d'un OCDE du Pacifique, l'intérêt de créer une union douanière ou même éventuellement une version Pacifique de la Communauté européenne.

Certes, les problèmes ne manquent pas dans la région : l'écart économique se creuse entre les pays de l'ASEAN et le développement institutionnel de cette organisation se fait plus lentement que prévu; des conflits raciaux ressurgissent en Malaisie et à Singapour; l'écrasante présence financière et industrielle du Japon demande la création d'un contrepoids au moment où les Etats-Unis connaissent un déclin relatif. Néanmoins, un vent de dynamisme y souffle et tous les pays y trouvent leur avantage.

Pendant ce temps, une sous-région de cet ensemble, celle de l'Asie du Nord-Est entourant la mer du Japon, est largement restée à l'écart du dynamisme de ce grand courant d'échange. 
Elle n'est pas encore arrivée à exploiter la complémentarité des pays et zones qui la composent et en font des partenaires naturels pour développer des projets de coopération régionale.

Cependant, l'évolution récente de la situation politique internationale transforme les perspectives de cette zone englobant les trois régions du Nord-Est de la Chine (Heilongjiang, Jilin et Liaoning), la Sibérie et l'Extrême-Orient soviétique, les deux Corées et le Japon.

Sa superficie représente $20 \%$ de l'Asie; la population est d'environ 300 millions et le PNB de plus de 3.000 milliards de $\$$. Il s'agit d'une zone qui si elle ne regroupe que $10 \%$ de la population asiatique produit environ $70 \%$ de son PNB.

\section{L'évolution de la situation internationale}

Le passé des pays qui la compose ainsi que les divisions résultant de la guerre froide ont freiné considérablement les contacts ces quarante dernières années.

A l'époque contemporaine, ce n'est qu'à la fin des années 70 que la Chine a commencé à se considérer comme un membre de la communauté du Pacifique. Dans la période de l'après-maoisme, un tournant important a été l'établissement en 1980 d'un institut de recherche sur l'économie des pays de l'Asie-Pacifique à l'Académie des Sciences Sociales. Cet institut a été le forum permettant de présenter des propositions prônant une plus grande implication de la Chine dans la région. Une coopération dans le Pacifique n'était plus nécessairement centrée sur la lutte entre les Etats-Unis et le Japon pour la suprématie régionale mais le moyen d'affirmer et défendre les intérêts du pays. L'objectif de modernisation de l'économie prôné par les pragmatiques au pouvoir à Pékin supposant l'accès à la technologie, aux biens d'équipements et aux devises 
permettant de les acheter, une collaboration devenait d'autant plus nécessaire. De plus, si la crainte d'un hégémonisme chinois n'est pas absente des esprits dans une perspective à long terme, la préoccupation actuelle majeure des Etats-Unis et des pays du Sud-Est asiatique est surtout de contenir la montée en puissance du Japon. Ils ont donc encouragé l'entrée de la Chine dans le cercle pour lui faire contrepoids.

Les Chinois ont longtemps considéré la politique Est-Asiatique japonaise comme déterminée par les intérêts de sécurité américains dans la région et, parallèlement, par la concurrence avec les Etats-Unis et le besoin d'assurer sa sécurité par des moyens économiques. L'échange de capital contre des matières premières maintenait des liens permanents de subordination dans le cadre d'un système dont le centre était au Japon.

En fait, l'évolution économique de la région, dont le Japon est un acteur important, certes, mais pas unique a rendu possible une plus grande intégration dont la Chine pourrait largement profiter. Les Nouveaux Pays Industrialisés (Hong-Kong, Taiwan, Singapour et la Corée du Sud) ont dépassé depuis longtemps le stade d'assembleurs de produits japonais de basse et moyenne technologie destinés à l'exportation vers l'Occident. Ils sont devenus fabricants de produits finis ou de composants de produits de haute technologie qu'ils exportent eux-mêmes au Japon, en Occident ou, de plus en plus, sur les marchés locaux. Si les investissements japonais y restent importants, les fabricants de produits à basse et moyenne technologie ont cédé la place à des fabrications japonaises de haute technologie ou des services de la finance, de l'immobilier, de la distribution et des loisirs.

La Chine est consciente qu'elle ne peut rester en dehors de ce courant. Le marché japonais s'ouvre aux produits 
manufacturés asiatiques et les fabricants japonais de produits de masse délocalisent vers des pays à plus bas coûts salariaux. En même temps, les nouveaux pays industrialisés deviennent eux-mêmes des investisseurs importants.

Les Chinois demeurent extrêmement méfiants quant aux intentions japonaises. Ils ne sont pas satisfaits de l'ampleur des investissements, ni de la faiblesse des importations japonaises ni des réticences japonaises à livrer leur technologie. Néanmoins, le Japon est leur deuxième partenaire commercial avec un volume d'affaires global d'environ 20 milliards de \$. Il a un programme d'aide officielle dont la Chine est un des principaux bénéficiaires. Le plus important contributeur aux fonds de la Banque Asiatique de Développement, il a été le premier des grands pays industrialisés à relâcher les restrictions après Tien An Men. Il est resté très prudent quant aux contacts à haut niveau mais a obtenu au sommet de Houston l'accord tacite de ses partenaires de pouvoir débloquer les prêts prévus pour un total de 6 milliards de $\$$.

Si la Chine continue à critiquer le Japon pour ses actions de guerre comme pour sa politique actuelle, elle sait très bien qu'il est un élément irremplaçable de sa politique de développement.

Pour ce qui est des relations avec l'URSS, la visite du président Gorbatchev en mai 1989 a mis un point d'orgue à une normalisation commerciale entamée depuis le début des années 80. Les échanges sino-soviétiques ont progressé de $47 \%$ en 1989 et le commerce frontalier a même triplé pendant la même période. Des contrats d'importation de matériel militaire et de biens d'équipement soviétiques sont négociés actuellement et le potentiel de croissance des échanges est très important.

La normalisation des relations sino-soviétiques a eu des conséquences importantes sur la situation politique et 
économique de la péninsule coréenne. Elle a tout d'abord permis la prise de contact de la Chine avec la Corée du Sud. Différentes raisons peuvent expliquer le rapprochement de ses deux pays : l'aspect économique est bien sûr primordial. La Corée du Sud est un trait d'union avec l'Asie du Sud-Est. C'est devenu aussi un investisseur non négligeable qui dispose de capitaux et d'une technologie moins chère et souvent mieux adaptée que celle du Japon aux besoins de la Chine. En 1989, les échanges commerciaux entre les deux pays se sont élevés à 3 milliards de $\$$. Une représentation commerciale faisant office de consulat a été ouverte dans les deux pays ouvrant la voie a l'établissement à terme de relations économiques normales.

Ensuite, dans le cadre du développement global de la région de l'Est asiatique, il existe une possibilité grandissante d'émergence d'un pôle de développement dont les points d'ancrage seraient Tokyo, Pusan, Dairen et Vladivostok. Il aurait comme hinterland économique la Sibérie, la Mongolie et la péninsule coréenne et ignorerait largement la Chine. C'est sans doute une des raisons de la volonté d'attirer la Corée du Sud dans l'arc côtier centré sur Shanghai que la Chine veut développer en priorité.

Il est évident que l'aide financière, technologique et d'ouverture de marchés que peut apporter la Corée du Sud est bien supérieur à ce qui peut être retiré des relations avec la Corée du Nord. Il s'agit néanmoins d'une situation délicate vu la profondeur des liens communs unissant la Chine et ce dernier pays. On observe que malgré les pressions nord-coréennes, la position chinoise s'infléchit au fil du temps. Elle s'oriente vers l'établissement progressif de relations diplomatiques équivalentes avec les deux régimes. Les prises de position de hauts responsables chinois à de multiples reprises ne laissent planer aucun doute sur les intentions chinoises à cet égard. 
La volonté d'être une nation commercialement active dans le Pacifique est également une préoccupation nouvelle pour l'URSS. Elle a été affirmée clairement dans les déclarations de Gorbatchev à Vladivostok en 1986 et à Krasnoyark en 1988. Cela suivait des années de reproche à l'égard d'une Communauté du Pacifique jugée agressive à son encontre et encourageant sa division avec la Chine. Le développement de la Sibérie et de la région d'Extrême-orient est un des objectifs majeurs des réformes entreprises depuis 1985. Il est clair à l'esprit des dirigeants soviétiques que ce ne peut se faire qu'en développant des relations avec le Japon, les nouveaux pays industrialisés et les pays de l'ASEAN. Bien que le Japon soit le quatrième partenaire capitaliste de l'URSS, les échanges commerciaux ne sont pas très importants : environ 6 milliards de $\$$ l'an. Ils ont d'ailleurs baissé de $6 \%$ pendant les six premiers mois de 1990 à cause des problèmes de paiement de plus en plus aigus de l'URSS. De même, le Japon reste un investisseur modeste : 33 joint ventures avec des partenaires soviétiques, la plupart dans l'exploitation forestière et la pêche.

Le paysage devrait néanmoins changer avec la visite au Printemps 91 de Gorbatchev au Japon. Le problème territorial ne sera sans doute pas entièrement réglé. Il est vain de croire que le Japon va retrouver sa souveraineté sur l'ensemble des Kouriles à cette occasion si jamais il la retrouve un jour. Cependant, jamais le climat n'a été aussi propice à un compromis, nécessaire à plus d'un titre pour les deux pays. Le Japon se rend compte qu'il se doit de participer activement au redressement des pays de l'Est. Un échec signifierait un isolement croissant dans une période de détérioration des relations avec les Etats-Unis et profiterait à l'extrême droite japonaise. Ce serait un coup sévère pour la diplomatie japonaise et sèmerait un nouveau doute sur ses capacités de régler des problèmes diplomatiques complexes. D'autre part, à l'exception de l'Allemagne, le Japon est sans doute mieux placé 
que la plupart des pays occidentaux pour aider l'URSS à développer ses ressources naturelles en Extrême-Orient et ailleurs. Or l'absence d'un règlement politique freine l'action de ses groupes industriels et financiers à un moment crucial du processus de réforme en URSS.

Malgré une volonté de compromis exprimée officiellement des deux côtés, l'affaire n'est pas du tout jouée : au Japon, l'opposition de la haute administration du Ministère des Affaires Etrangères à toute concession à l'URSS contraste avec la position de plus en plus entreprenante des leaders du Parti Libéral Démocrate qui se succèdent à Moscou en vue de réussir une percée payante politiquement. Tout compromis demandera un délicat arbitrage politique. De son côté, le gouvernement soviétique fait face à des forces centrifuges de plus en plus puissantes qui n'encouragent pas à un compromis territorial quelque soient les nécessités.

En ce qui concerne les relations URSS-Corée du Sud, les événements se sont succédés plus rapidement que prévu. Pour les deux gouvernements, la motivation est double : la préoccupation de sécurité ne quitte jamais les esprits à Séoul. L'ouverture vers le Nord est considérée comme cruciale pour la stabilisation de la péninsule. Créer une voie vers Pyong Yang via Moscou est la pierre angulaire de la politique du Président Roh Tae Woo. D'un autre côté, l'accélération des échanges commerciaux qui pourraient s'élever à plus de 10 milliards de \$ à 5 ans, pousse à une normalisation rapide. La Corée du Sud est déjà un fournisseur important de biens de consommation tels qu'appareils électriques et ordinateurs et un gros acheteur de bois et charbon soviétique. La communauté d'affaires coréenne désire approfondir ces liens. Les frictions commerciales croissantes avec les Etats-Unis forcent les groupes industriels coréens à chercher de nouveaux marchés. De grands projets sont envisagés comme par exemple celui du groupe Hyundai 
d'importation de gaz et de pétrole soviétiques via un pipeline partant de Vladivostok et passant par la Corée du Nord. En même temps, cette politique permet de devancer les groupes japonais bloqués par les négociations territoriales de leur gouvernement et impliqués beaucoup plus que la Corée dans le système de concertation politique entre pays développés sur l'opportunité et la manière de considérer une aide à l'URSS. Le Japon ne tient pas à se singulariser à cet égard vu ses relations avec les Etats-Unis, ce qui freine le développement des relations commerciales.

Pour l'URSS aussi, la stabilité de la péninsule est primordiale. L'ouverture vers Séoul permet d'accentuer la pression sur la Corée du Nord pour la forcer à une réduction militaire et à un contrôle de son programme nucléaire. Il s'agit d'une partie difficile à jouer avec un allié de longue date. Un sentiment d'isolement trop intense pourrait tout aussi bien avoir l'effet de durcir la position de Pyong Yang et pousser la Corée du Nord à l'aventure.

Parallèlement, les Soviétiques ont présenté en 1990 aux groupes industriels coréens une liste indicative de projets allant d'un complexe pétrochimique à des exploitations de ressources énergétiques. Il s'agit de projets de très grande envergure nécessitant un financement complexe et une stratégie de développement à long terme. La réaction coréenne a été d'observer qu'ils dépassent souvent les possibilités de la Corée du Sud ou tout au moins les limites de ce que les groupes industriels envisagent de consacrer à l'URSS. Si une collaboration économique importante est certainement possible entre les deux pays, aucun n'est dupe des limites de celle-ci : après une période d'euphorie au cours de laquelle de très grands projets avaient été envisagés, les Coréens sont redevenus plus réalistes quant aux possibilités offertes par l'URSS. Ils sont conscients de leurs faiblesses industrielles et 
financières et savent que les Soviétiques sont sans illusion à ce propos. L'action économique vers la Corée est en grande partie un aiguillon utilisé vis-à-vis des groupes japonais au moment où une ouverture est possible dans les négociations territoriales qui pourra enfin permettre leur nécessaire participation aux projets sibériens.

De l'autre côté de la péninsule, progressivement lâché par un allié chinois en sérieuse difficulté économique qui ne peut plus retarder son ouverture vers la Corée du Sud et, de toute façon, ne peut pas grand chose pour lui en termes militaires, technologique et approvisionnement énergétique; de plus, brutalement ramené à la réalité par les rencontres entre Roh Tae Woo et Gorbatchev, la Corée du Nord n'a jamais été aussi isolée. Pour rompre cet isolement, elle multiplie les ouvertures en Asie du Sud-Est et en Australie. Une approche des EtatsUnis a même été entreprise avec la restitution symbolique de restes de soldats américains.

Le Japon répond déjà à l'ouverture entre Séoul et Moscou par une tentative de normalisation de ses relations avec la Corée du Nord. Les échanges commerciaux représentent 1/60ème de ceux avec la Corée du Sud. Néanmoins, le Japon est le troisième partenaire commercial de Pyong Yang grâce au soutien de sa communité coréenne. La présence de joint-venture textiles créées par ces Coréens du Japon permet même à la Corée du Nord d'avoir un surplus commercial de 78 millions de $\$$ avec ce pays.

Cela ne compense que très peu le déficit commercial croissant, essentiellement avec l'URSS et la Chine. Jusqu'à présent, il était automatiquement converti en dette à long terme, ce qui représentait une forme d'assistance financière en gage de l'amitié entre pays socialistes. A la fin de 1989, la Corée du Nord devait à l'URSS environ 3,6 milliards de \$. La 
conjoncture actuelle n'autorise plus de telles largesses et le fardeau que représente pour des économies en difficulté comme celles de la Chine et de l'URSS un pays dont l'importance stratégique a beaucoup baissé, oblige la Corée du Nord à s'ouvrir.

Malgré les risques que représentent une implication dans les affaires coréennes d'un pays au lourd passé colonial comme le Japon, tout semble indiquer que celui-ci est prêt à jouer un rôle croissant dans le redressement de l'économie nord-coréenne. Le principe de négociations sur les compensations pour préjudices subis par les Coréens pendant l'ère coloniale et la seconde guerre est accepté par le gouvernement japonais. Cela indique une volonté de normalisation des relations qui devrait commencer par l'ouverture d'un bureau de liaison dès cette année. Ce mouvement vers une reconnaissance croisée des deux Corées fait partie d'un accord négocié globalement entre les Occidentaux (incluant le Japon, la Chine et l'URSS). Cependant, la Corée du Sud est très préoccupée par ce renforcement rapide des relations entre le Japon et la Corée du Nord qui améliore la position diplomatique de cette dernière. $\mathrm{La}$ tension pourrait de ce fait augmenter pour un moment dans la péninsule. Malgré les prises de contact avec le Japon, la Corée du Nord demeure opposée à l'idée d'une reconnaissance croisée qui consacrerait à ses yeux la division du pays. Il est donc hâtif de croire que l'éventualité de l'établissement de relations diplomatiques avec le Japon va pousser la Corée du Nord à changer sa position.

Les relations intracoréennes restent d'ailleurs le point noir d'un ensemble Est-Asiatique beaucoup plus ouvert qu'il y a quelques années. Si la Corée du Nord cherche à créer des ouvertures dans le monde capitaliste, le dialogue entre Coréens ne progresse guère. Ayant établi des canaux de dialogue indépendants avec Moscou et Pékin et le regroupement des 
forces conservatrices lui donnant une majorité confortable, le gouvernement Sud-coréen est confiant de pouvoir gérer la détente avec le Nord. Cependant, la rencontre de leurs deux Premiers Ministres au début septembre a montré l'importance de l'écart qui existe toujours entre les deux régimes. Malgré tous ses problèmes économiques et son isolement diplomatique, la position de la Corée du Nord ne change pas. La péninsule doit être libérée du joug impérialiste par son régime, seul représentant légitime du peuple coréen. Toute nouvelle de mouvement social en Corée du Sud, par ailleurs inhérent au passage d'une société autoritaire à une démocratie, est considérée comme l'amorce d'un changement révolutionnaire. L'immobilisme interne du régime face à une Corée du Sud ayant effectivement à faire face à des probèmes sociaux graves mais progressant sur la voie de la démocratisation, renforce la perception qu'une crise majeure n'est pas à exclure débouchant sur un changement violent.

Le développement de la coopération dans l'Asie du Nord-Est

Malgré le caractère encore inachevé de beaucoup d'initiatives diplomatiques dans les cinq pays concernés, les conditions n'ont certainement jamais été meilleures pour le développement d'une collaboration régionale multilatérale.

Certaines remarques peuvent être faites sur les caractéristiques de la région par rapport à la situation économique ayant conduit à l'accord de libre-échange entre les Etats-Unis et le Canada, celui prôné par l'APEC à Canberra ou encore la Communauté européenne.

Par rapport aux ensembles précités, la région se caractérise par une très grande différentiation de l'allocation des facteurs de production. Il y a les ressources de la pêche, le charbon, le pétrole et le bois de l'Extrême-Orient soviétique; les ressources 
agricoles de la Chine du Nord-Est : haricot, maïs, autres légumes et fruits; l'or, le tungstène, le fer et la main-d'oeuvre nord-coréenne; la capacité de production de biens d'équipement et de consommation, la puissance financière et l'expertise de la gestion de grands projets de la Corée du Sud et surtout du Japon.

Les échanges commerciaux entre les pays ont une nature verticale : ressources naturelles contre produits manufacturés, produits à haute intensité de main-d'oeuvre contre biens de haute technologie. Il est peu probable que cette situation change dans un avenir proche. Dans son plan de développement de l'Extrême-Orient publié en 1987, le gouvernement soviétique met l'accent sur le développement de secteurs à forte intensité capitalistique et technologique. Cela semble peu réaliste alors que l'avantage comparatif de la région est dans le développement des ressources naturelles telles que métaux non-ferreux, charbon, pétrole, gaz naturel, bois et produits de la mer.

Le Nord-Est asiatique ne peut pas non plus devenir une zone fermée. Deux membres clés, le Japon et la Corée du Sud ont des relations commerciales importantes avec les Etats-Unis, l'Europe et l'Asie du Sud-Est. Dans le cas de la Chine et de l'URSS, seule une partie du territoire est concernée. Pour les cinq pays, les relations en Asie du Nord-Est ne représentent qu'une partie relativement faible de leurs relations commerciales extérieures. En ne se limitant pas stricto sensu à cette région, le total des échanges commerciaux entre eux s'est élevé à 94,5 milliards de $\$$ en 1989. Pour ce qui concerne le degré d'implication de chacun : la Corée du Nord y réalise $65 \%$ de ses échanges commerciaux; la Chine et la Corée du Sud, $30 \%$; le Japon et l'URSS, moins de $10 \%$. Le commerce bilatéral entre l'URSS et la Corée du Nord représente $20 \%$ de leurs échanges commerciaux globaux; entre la Chine et le Japon, la Corée du 
Sud et le Japon, la Chine et la Corée du Nord, le chiffre est d'environ $10 \%$; pour les relations entre l'URSS et la Chine et celles entre l'URSS et la Corée du Sud, il est de $3 \%$; enfin, pour ce qui est de l'URSS et du Japon, il est de $2 \%$.

\section{Le développement de points d'ancrage}

Pour développer une cohésion régionale dans une région aussi hétérogène, il faut trouver des possibilités de collaboration sur des projets concrets englobant plusieurs pays.

Un de ceux-ci peut être le développement de l'ExtrêmeOrient soviétique. La situation économique y reste précaire : la base industrielle est faible, les zones de développement progressent très peu par manque de financement, les ressources de la région sont toujours drainées vers le centre et les autorités ne jouissent pas encore d'une autonomie réelle suffisante malgré la "perestroika". La situation pourrait changer avec l'arrivée de Boris Eltsine et sa volonté de décentralisation depuis son élection à la présidence de la république de Russie. Les autorités de Sakhaline ont proclamé unilatéralement l'ile zone économique spéciale. En conformité avec la nouvelle loi sur le commerce extérieur votée par le Soviet suprême de la république de Russie, ce statut a été accordé à la région maritime autour de Nakhodka et au district de Chita à l'Est du lac Baikal.

Pour satisfaire aux objectifs du plan, des investissements de l'ordre de 230 milliards de roubles seront nécessaires d'ici l'an 2000. Cependant, comme la croissance de l'économie soviétique est inférieure aux prévisions et que le 13ème plan quinquennal donne la priorité au développement technologique des entreprises de la partie européenne de l'URSS, il reste peu de ressources pour les grands projets de l'Extrême-Orient. Comme la base économique de la région reste très faible, il est 
peu probable qu'elle puisse générer plus de $20 \%$ de la somme. Cela signifie que le rendement des investissements sera de la plus grande importance et que la sélection des projets doit être plus sévère qu'auparavant. Un des grands obstacles au développement de l'Extrême-Orient soviétique est le manque d'infrastructure aérienne, maritime, routière et ferrovière. C'est pourquoi ce secteur est considéré comme prioritaire par le plan. $\mathrm{Vu}$ les conditions climatiques et géographiques, tout projet nécessite une expertise technique et des moyens financiers dont ne dispose pas l'URSS.

Ces obstacles expliquent pourquoi la région s'efforce de participer à la division internationale du travail, particulièrement avec les pays de l'Asie Pacifique. A ce jour, seul un très petit nombre de joint-ventures a été constitué dans l'Extrême-Orient soviétique : 26 sur un total d'environ 800 pour l'ensemble de l'URSS. Parmi ces 26, le Japon est le premier partenaire avec 9 , suivi des Etats-Unis avec 6, la Corée du Nord avec 3, la Chine avec 2 et Singapour et la Nouvelle-Zélande avec 1 .

Dans le domaine de l'énergie, une joint-venture au capital de 100 millions de \$ a été constituée par Mac Dermott (EU), Neste (Finlande), Mitsui Corporation et Mitsubishi Corporation (Japon) et la banque anglaise Morgan Grenfell pour la construction et l'exploitation d'un combinat pétrochimique dans l'Ouest de la Sibérie. Un groupe de firmes sud-coréennes étudie la faisabilité de l'exploitation commerciale des grandes centrales électriques au charbon de la Sibérie et de la région d'Extrême-Orient. Les projets de développement de l'exploitation des gisements de gaz et de pétrole de la plateforme continentale de Sakhaline qui avaient mené à un accord symbole de la collaboration sovietojaponaise, sont renégociés depuis un an après avoir été gelés pendant plus de 10 ans. 
Dans le domaine de l'exploitation forestière, il y a deux exemples de joint-ventures soviéto-japonaises se développant de manière satisfaisante. Actuellement, le groupe sud-coréen Hyundai et un groupe forestier local développent un projet d'exploitation des forêts sibériennes dans la région de Svetlaya, $700 \mathrm{~km}$ au Nord-Est de Nakhodka. Le projet est d'envergure et nécessitera l'emploi d'un grand nombre de travailleurs nordcoréens et chinois. Déjà, Hyundai a approché Mitsubishi Corporation et d'autres tradings japonaises pour développer le marché japonais.

Un des grands obstacles au développement du commerce entre les trois régions chinoises du Nord-Est et l'ExtrêmeOrient soviétique est la quasi absence de réseau de transport. On peut parcourir les rives de l'Amour et de l'Oussouri sur des centaines de kilomètres sans pouvoir traverser la frontière faute de pont.

Il y a maintenant une ligne aérienne directe entre Harbin (Chine) et Vladivostok et une autre est prévue entre Pusan (Corée du Sud) et Vladivostok. Une représentation commerciale sud-coréenne est déjà installée dans cette ville. Une voie de chemin de fer passant sur un pont est aussi prévue entre la ville soviétique de Bulagoveschenskov et la ville chinoise de Heichuan sur le fleuve Amour.

Dans le domaine du tourisme et des services, il y a 5 jointventures sovieto-japonaises dans l'hôtellerie. Un projet de ce type avec collaboration triangulaire soviéto-nippo-chinoise est même à l'étude ainsi que l'ouverture simultanée de magasins soviéto-chinois de produits textiles et de cuir à Vladivostok et Heichuan.

Les projets de développement de l'Extrême-Orient soviétique utilisent aussi une abondante main-d'oeuvre 
asiatique : environ 100.000 Nord-coréens et Chinois travaillent dans l'exploitation forestière dans la région de Khabarovsk et le long de l'Amour. Ils sont aussi employés de plus en plus dans l'industrie.

Un exemple de collaboration soviéto-japonaise a été l'aménagement du port de Nakhodka qui permettra une amélioration sensible du transport dans la région lorsqu'il sera tout à fait opérationnel et relié par train avec le port de Vostochinei à $30 \mathrm{~km}$ au Nord. Il pourra alors transporter 25 millions de tonnes de marchandises par an. Ce sera l'un des débouchés les plus importants de la région.

S'il n'est pas encore concrétisé, un autre projet pourrait devenir un exemple de collaboration entre pays riverains. Il s'agit de la construction d'un port international en territoire chinois à l'embouchure du fleuve Tumen qui se jette dans la mer du Japon. Le fleuve coule entre la région chinoise de Jilin et la Corée du Nord. L'embouchure a comme caractéristique d'être à la frontière de trois pays : l'URSS, la Corée du Nord et la Chine. A gauche est située la zone économique spéciale soviétique de Poset. A droite, un peu en retrait se trouvent les ports nord-coréens de Chongjin et Rajin que le gouvernement nord-coréen veut agrandir pour en faire les entrées d'une zone franche. Le territoire chinois s'arrête à environ $15 \mathrm{~km}$ de l'embouchure. La Chine n'ayant plus eu de droit de navigation sur le fleuve depuis 1938, les territoires du Nord-Est n'ont pas de débouché sur la mer du Japon. Pour commercer avec l'extérieur, ils doivent passer par le Sud et utiliser le port de Dairen. C'est un grand handicap pour la région de Jilin et plus particulièrement pour la région autonome coréenne de Yanji.

La détente dans les relations sino-soviétiques a permis d'obtenir un droit de passage. Il a été accordé lors de la visite de 
Gorbatchev à Pékin en 1989. Avec la Corée du Nord, les négociations sont en cours.

La construction du port et le dragage des rives nécessiteront un financement principalement japonais ainsi que l'expertise de firmes de construction de ce pays. Les liaisons avec la côte ouest japonaise, principalement les préfectures de Toyama et Niigata seront beaucoup facilitées de même que celles avec Pusan.

La zone autonome coréenne qui est au centre du projet est aussi une région dont le rayonnement international est susceptible de profiter des bienfaits de la coopération. Sa capitale accueille un des plus grands marchés agricoles de la région. A Tumen se trouve le siège de la plus grande firme privée de la région de Jilin. Elle est donc en liaison étroite avec l'hinterland chinois.

Ce projet est d'autant plus intéressant qu'il ferait renaître un point de passage important entre le Japon et la Chine utilisé par les missions diplomatiques des 2 pays du 8ème au 10ème siècles.

\section{Conclusion}

Après des années de relative négligence, les firmes et gouvernements régionaux japonais sont de nouveaux beaucoup plus intéressés par une zone de développement autour de la mer du Japon. C'est le cas aussi des firmes sud-coréennes qui cherchent de nouveaux marchés ainsi que des sources alternatives de matières premières et qui pourraient utiliser l'abondante main-d'oeuvre coréenne dans la région.

C'est sans doute l'attitude des deux pays les plus développés de la région qui dans un premier temps va être le facteur clé du développement d'une zone qui pourrait connaître 
un taux de croissance très élevé dans les prochaines années pour peu que les ouvertures politiques actuelles se concrétisent. Les projets concrets demeurent relativement peu nombreux et ce sont souvent le Japon et la Corée du Sud qui y impliquent les autres pays. Cela ne devrait cependant pas toujours être le cas vu le développement grandissant de l'esprit d'entreprise des Soviétiques et des Chinois qui ont compris les limites de l'aide que pouvait leur apporter leur gouvernement central. Les trois régions du Nord-Est de la Chine sont un fardeau pour Pékin et le retard de leur développement par rapport aux régions du Sud et du centre est difficile à combler. De son côté, l'ExtrêmeOrient soviétique ne peut plus compter beaucoup sur les investissements du gouvernement central. Le niveau de complémentarité entre les trois régions agricoles et d'élevage de la Chine du Nord-Est et celles productrices de bois, de ciment, d'engrais et de minerais de l'Extrême-Orient soviétique est très grand. Cela permet des activités de troc à grande échelle économisant des devises aux deux pays.

Les premières joint-ventures sino-soviétiques sont modestes et largement concentrées dans les services. Cependant, il existe aussi de grands projets de développement de l'exploitation des ressources hydro-électriques, d'irrigation, de pêche, de navigation et de production d'eau potable du fleuve Amour. De même, la modernisation des usines de fabrication soviétique des années 50 fait l'objet de nombreuses discussions.

Certes, les problèmes demeurent. Le faible niveau de la technologie soviétique fait craindre aux Chinois que l'appel fait aux techniciens soviétiques pour moderniser les équipements vieillis ne permette pas à la Chine de combler son retard technologique, bien au contraire. Cela renforce d'autant plus le besoin de collaboration avec la Corée du Sud et le Japon. De même, le caractère encore bureaucratique des Soviétiques dans 
leur gestion du commerce frontalier ainsi que le manque d'autonomie des autorités locales constituent des freins au développement des échanges. Dans le cadre de leurs relations avec le Japon et la Corée du Sud, les Soviétiques mettent encore trop souvent l'accent sur le développement de grands projets. Cela ne cadre pas souvent avec l'intérêt des Japonais et Coréens pour des opérations de plus petites tailles pouvant être financées plus facilement et présentant moins de risque.

Dans cette dynamique d'échanges, seule la Corée du Nord reste à la traîne. Elle est encore peu impliquée dans les projets de joint-ventures sauf sur une très petite échelle avec la communauté coréenne du Japon et la structure de son commerce avec les pays de la région change très peu. Les changements de politique de ses partenaires devraient cependant lui permettre de s'intégrer progressivement dans la dynamique régionale. L'avantage évident d'une participation plus active devrait alors assouplir sa position au fil du temps. 


\section{Bibliographie}

- Inteflo $1989 \mathrm{~N}^{\circ} .9$.

- Jiji Nenkan 1990.

- The Europa Year Book 1989.

- Chosen Nenpo 1989. 10. 14.

- Keizai Sokuho $\mathrm{N}^{\circ} .812$.

- Asahi Shimbun 199023 février.

- The China Business Review 1990 janvier-février.

- Far Eastern Economic Review 1990 : 14 juin, 9, 23, 30 août 20 septembre.

- NIRA Kenkyu Gyosho N. 90066.

- The Economist 15 juillet 1989.

- The Japan Economic Journal : 8 août 1989, 5, 12 septembre 1990.

- Soren-Toyo Keizai to Nihon (Relations du Japon avec l'URSS et les pays de l'Est européen) avril 1990. Editeur Keizai Chosakai. 


\section{SUMMARY}

Compared to the very fast development of South-East Asia and the multiplicity of regional collaboration projects, the region bordering the Japan Sea had a rather slow development for the last 20 years and very few common projects have been developed despite high complementarity in factors endownment.

The detente in the East-West relations has progressively transformed the political climate leading to quite unexpected rapproachment between socialist and capitalist countries but also among socialist countries in the region. If nothing definitive has been achieved so far, it seems that even the untractable situation on the Korean Peninsula could very well change in a foreseeable future. It is only the beginning of a new era but this should bring development of more and more projects involving several countries of the area. 